\title{
WEAK TYPE $(1,1)$ ESTIMATES FOR A CLASS OF DISCRETE ROUGH MAXIMAL FUNCTIONS
}

\author{
Roman Urban and JaceK ZienKieWicZ
}

ABStract. We prove weak type $(1,1)$ estimate for the maximal function associated with the sequence $\left[m^{\alpha}\right], 1<\alpha<1+\frac{1}{1000}$. As a consequence, the sequence $\left[m^{\alpha}\right]$ is universally $L^{1}$-good.

\section{Introduction and statement of the result}

Let

$$
\mathcal{M}^{*} f(x)=\sup _{M>0} \frac{1}{M} \sum_{0<m<M} f\left(x-\left[m^{\alpha}\right]\right), x \in \mathbb{Z} .
$$

The aim of this note is to prove the weak type $(1,1)$ of the maximal function $\mathcal{M}^{*}$ for $1<\alpha<1+\frac{1}{1000}$. Thus we provide a counterexample of arithmetic set type to the conjecture of J. Rosenblatt and M. Wierdl, see [4]. We use an approach similar to those of M. Christ [5], see also [8, 11]. We reduce the problem of the weak type $(1,1)$ of $\mathcal{M}^{*}$ to the regularity estimates for the convolution of a certain measure $\mu_{M}$ supported by the sequence $\left[\mathrm{m}^{\alpha}\right]$ and its reflection $\tilde{\mu}_{M}$. This is closely connected to the problem of representation of a given integer as a difference of two numbers of the form $\left[m^{\alpha}\right]$. In order to obtain necessary estimates, we use B. I. Segals approach, [7], [9], see also [6]. The $\ell^{p}(1<p \leq \infty)$ boundedness of the maximal function $\mathcal{M}^{*}$ has been established in [1,3] and [2].

Our main result is the following

Theorem 1.1. Let $1<\alpha<1+\frac{1}{1000}$. Then the operator $\mathcal{M}^{*}$ defined above is of weak type $(1,1)$.

Recall that a sequence of integers $\left\{a_{n}\right\}_{n \in \mathbb{N}}$ is universally $L^{1}$-good if the following property holds: for any measure preserving ergodic flow $\left\{T^{s}\right\}_{s \in \mathbb{Z}}$ on any probability space $(\Omega, \mathcal{F}, \mu)$ and $f \in L^{1}(\Omega, \mu)$ the averages

$$
\frac{1}{N} \sum_{n \leq N} f \circ T^{a_{n}} \rightarrow \int f d \mu
$$

$\mu$-a.e. as $N \rightarrow \infty$.

Received by the editors March 9, 2006.

2000 Mathematics Subject Classification. 42B25, 11P05.

Key words and phrases. Rough operators, Maximal function, Calderón-Zygmund theory.

Research supported in part by RTN Harmonic Analysis and Related Problems contract HPRNCT-2001-00273-HARP, the European Commission Marie Curie Host Fellowship for the Transfer of Knowledge "Harmonic Analysis, Nonlinear Analysis and Probability" MTKD-CT-2004-013389. The first author was also supported by the MNiSW research grant N201 012 31/1020. 
Corollary 1.2. The sequence $\left[m^{\alpha}\right], 1<\alpha<1+\frac{1}{1000}$ is universally $L^{1}$-good.

Proof. By now, the classical argument can be found in [1].

Remark 1.3. The range of $1<\alpha<1+\frac{1}{1000}$ can be improved by the method used in the paper.

\section{Some lemmas}

For a fixed integer $Q \geq 1$, denote $x_{P}=x-\frac{P}{Q}, P=0,1, \ldots, Q-1$. In our application $Q$ will be $M^{\frac{1}{1000}}$.

Lemma 2.1. Let $M \leq m \leq 2 M$, and $x_{P} \geq M$, and

$$
f_{P}(m)=j_{1}\left(x_{P}+m^{\alpha}\right)^{1 / \alpha}+j_{2} m^{\alpha},
$$

where $\left|j_{1}\right| \leq M^{\frac{1}{100}+\alpha-1}$ and $\left|j_{2}\right| \leq M^{\frac{1}{100}}$. Then there exist $m_{0} \in(M, 2 M)$ such that for

$$
\left|m-m_{0}\right| \geq M^{\frac{99}{100}}
$$

we have

$$
c_{\alpha} M^{-\frac{103}{100}} \leq f_{P}^{\prime \prime}(m) \leq C_{\alpha} M^{-\frac{98}{100}}
$$

Proof. Straightforward calculation shows that

$$
f_{P}^{\prime \prime}(m)=(\alpha-1) m^{\alpha-2}\left(\frac{x_{P} j_{1}}{\left(x_{P}+m^{\alpha}\right)^{2-\frac{1}{\alpha}}}+j_{2} \alpha\right)
$$

Denote $A(m)=\left(\frac{x_{P} j_{1}}{\left(x_{P}+m^{\alpha}\right)^{2-\frac{1}{\alpha}}}+j_{2} \alpha\right)$. Assume that for every $M \leq m \leq 2 M$ we have $|A(m)| \geq \frac{1}{100} M^{-\frac{3}{100}}$. Then $\left|f_{P}^{\prime \prime}(m)\right| \geq c_{\alpha} M^{-\frac{103}{100}}$ and the lower bound follows. Assume that $\left|A\left(m_{0}\right)\right| \leq \frac{1}{100} M^{-\frac{3}{100}}$ and observe that for $\left|x_{P}\right| \geq M$ and $\left|m_{0}-m\right| \geq M^{\frac{99}{100}}$ we have, using mean value theorem,

$$
\left|A(m)-A\left(m_{0}\right)\right|=\left|\frac{x_{P}}{\left(x_{P}+m^{\alpha}\right)^{2-\frac{1}{\alpha}}}-\frac{x_{P}}{\left(x_{P}+m_{0}^{\alpha}\right)^{2-\frac{1}{\alpha}}}\right| \geq \frac{1}{10} M^{-\frac{3}{100}} .
$$

Hence for $\left|m_{0}-m\right| \geq M^{\frac{99}{100}}$ we have $|A(m)| \geq \frac{1}{100} M^{-\frac{3}{100}}$. The lower bound for $f_{P}^{\prime \prime}(m)$ follows. Direct calculation easily shows the upper bound

$$
\left|f_{P}^{\prime \prime}(m)\right| \leq C_{\alpha} M^{-\frac{98}{100}}
$$

Corollary 2.3. Let $M^{\frac{1}{200}} \leq k \leq 2 M^{\frac{1}{200}}, \phi \in C_{c}^{\infty}(-4,4)$ and

$$
S\left(j_{1}, j_{2}\right)=\sum_{M^{1-\frac{1}{200}}} \sum_{k \leq m \leq M^{1-\frac{1}{200}}(k+1)} \phi\left(\frac{m}{M}\right) e^{2 \pi i f_{P}(m)} .
$$

Then, under the same assumptions as in Lemma 2.1, we have

$$
\left|S\left(j_{1}, j_{2}\right)\right| \leq C_{\alpha} M^{1-\frac{1}{200}} M^{-\frac{1}{200}}
$$

Proof. We use the following 
Theorem 2.4 (Van der Corput, [12]). Let $a, b, k$ be positive integer numbers such that $a<b, l \geq 2$. Let $f \in C^{l}([a, b])$. Denote $r=\inf _{x \in[a, b]}\left|f^{(l)}(x)\right|$ and $R=$ $\sup _{x \in[a, b]}\left|f^{(l)}(x)\right|$. Then

$$
\begin{aligned}
\left|\sum_{m=a}^{b} e^{f(m)}\right| & \\
& <21(b-a)\left(\left(\frac{r}{R^{2}}\right)^{-1 /(\kappa-2)}+\left(r(b-a)^{l}\right)^{-2 / \kappa}+\left(\frac{r(b-a)}{R}\right)^{-2 / \kappa}\right),
\end{aligned}
$$

where $\kappa=2^{l}$.

Then we take $\phi(t)=1$ for $0 \leq t \leq 2$, fix $k, j_{1}, j_{2}$ and apply the above theorem to estimate two sums $S\left(j_{1}, j_{2}\right)$ taken over the intervals

$$
\max \left\{m_{0}+M^{1-\frac{1}{100}}, M^{1-\frac{1}{200}} k\right\} \leq m \leq M^{1-\frac{1}{200}}(k+1)
$$

and

$$
M^{1-\frac{1}{200}} k \leq m \leq \min \left\{M^{1-\frac{1}{200}}(k+1), m_{0}-M^{\frac{99}{100}}\right\} .
$$

We apply the above theorem with $l=2, r \geq M^{-\frac{103}{100}}, R \leq M^{-\frac{98}{100}}, b-a=M^{1-\frac{1}{200}}$ and we see that the sum is bonded by $M^{1-\frac{1}{200}} M^{-\frac{1}{4}}$. Consequently, remember (2.2), we have $\left|S\left(j_{1}, j_{2}\right)\right| \leq C_{\alpha} M^{1-\frac{1}{200}} M^{-\frac{1}{200}}$. See [7].

The case of nonconstant $\phi$ follows in a standard way by Abel summation formula.

Lemma 2.5. Let, for a fixed $\varphi \in C_{c}^{\infty}(1,2)$ with $\int \varphi=1, \mu_{M}$ be a measure on $\mathbb{Z}$ defined as follows

$$
\mu_{M}(x)=\frac{1}{M} \sum_{m \in \mathbb{Z}} \delta_{0}\left(x-\left[m^{\alpha}\right]\right) \varphi\left(\frac{\left[m^{\alpha}\right]}{M^{\alpha}}\right),
$$

where $\delta_{0}$ stands for Dirac's delta. Then, for $\tilde{\mu}(x)=\mu(-x)$,

$$
\mu_{M} * \tilde{\mu}_{M}(x)=\rho_{M}(x)+O\left(M^{-\alpha-\frac{1}{1000}}\right),
$$

where $\rho_{M}(0)=M^{-1}$ and for $x \neq 0$ we have

$$
0 \leq \rho_{M}(x) \leq C M^{-\alpha} .
$$

Furthermore, for $x \geq C M$ and $x+h \geq C M$,

$$
\left|\rho_{M}(x+h)-\rho_{M}(x)\right| \leq C \frac{|h|}{M^{2 \alpha}} .
$$

Similar statement holds if $x \leq-C M$ and $x+h \leq-C M$.

Proof. We start with the proof of (2.6). Since $\mu_{m} * \tilde{\mu}_{m}$ is symmetric, it suffices to consider the case $x \geq C M$ and $x+h \geq C M$. We fix $Q=M^{\frac{1}{1000}}$, and define

$$
\begin{aligned}
u_{k} & =M^{1-\frac{1}{200}} k, \\
x_{P} & =x-\frac{P}{Q}, P=0,1, \ldots, Q-1, \\
A_{k} & =\frac{1}{\alpha\left(x_{P}+u_{k}^{\alpha}\right)^{\frac{\alpha-1}{\alpha}}} .
\end{aligned}
$$


Let $F$ be a $C^{\infty}$ function such that $0 \leq F \leq 1$ and

$$
F(x)= \begin{cases}1 & \text { if } x \leq 0 \\ 0 & \text { if } x \geq 1\end{cases}
$$

Define $\Psi_{k, P}^{\mathrm{u}}(x)$ as a periodic, with period 1, extension of

$$
\Psi_{k, P}^{\mathrm{u}}(x)= \begin{cases}F\left(\frac{Q}{A_{k}}\left(x-\frac{10 A_{k}}{Q}\right)\right) & \text { if } 0 \leq x \leq \frac{1}{2}, \\ F\left(\frac{Q}{A_{k}}\left(-A_{k}\left(1+\frac{10}{Q}\right)-x\right)\right) & \text { if }-\frac{1}{2} \leq x \leq 0 .\end{cases}
$$

Let $G \in C_{c}^{\infty}(-1,1)$ and $\sum_{s \in \mathbb{Z}} G(x-s) \equiv 1$. Define,

$$
\Psi_{P}(x)=\sum_{s \in \mathbb{Z}} G\left(Q\left(x_{P}+s\right)\right) \in C_{c}^{\infty}(\mathbb{T}) .
$$

It is easy to see that

$$
\sum_{P=0}^{Q-1} \Psi_{P}(x) \equiv 1
$$

Observe that by $(2.9)$,

$$
\begin{aligned}
& \mu_{M} * \tilde{\mu}_{M}(x) \leq C M^{-\alpha-1}+ \\
& \frac{1}{M^{2}} \sum_{P=0}^{Q-1} \sum_{k=M}^{2 M} \sum_{\substack{200 \\
\frac{1}{200}}}^{M^{1-\frac{1}{200}}(k+1)} \sum_{m=M^{1-\frac{1}{200}} k} 1_{A}\left(x+\left[m^{\alpha}\right]\right) \Psi_{P}\left(m^{\alpha}\right) \varphi\left(\frac{m^{\alpha}}{M^{\alpha}}\right) \varphi\left(\frac{x+m^{\alpha}}{M^{\alpha}}\right) \\
& \leq C M^{-\alpha-1}+I ;
\end{aligned}
$$

error term $C M^{-\alpha-1}$ appears because of replacing $\varphi\left(\frac{\left[m^{\alpha}\right]}{M^{\alpha}}\right)$ by $\varphi\left(\frac{m^{\alpha}}{M^{\alpha}}\right)$ and is easily estimated by Taylor's formula. We will prove the estimate

$$
\begin{aligned}
& I \leq \\
& \frac{1}{M^{2}} \sum_{P=0}^{Q-1} \sum_{k=M \frac{1}{200}}^{*} \sum_{m=M^{1-\frac{1}{200}} k}^{\frac{1}{200}-1} \Psi_{k, P}^{\mathrm{u}}\left(\left(x_{P}+m^{\alpha}\right)^{1 / \alpha}\right) \Psi_{P}\left(m^{\alpha}\right) \varphi\left(\frac{m^{\alpha}}{M^{\alpha}}\right) \varphi\left(\frac{x+m^{\alpha}}{M^{\alpha}}\right),
\end{aligned}
$$

where $A=\left\{\left[m^{\alpha}\right]: m \in \mathbb{N}\right\}$, and $*$ in the sign of summation above denotes that the first term with $P=0$ is taken two times: with $x_{P}=x$ and $x_{P}=x+1$. In order to prove (2.10) we need to show that the conditions (here $\|x\|=\min _{k \in \mathbb{Z}}|x-k|$, denotes the distance of $x$ to the nearest integer)

$$
\begin{gathered}
\left\|m^{\alpha}-\frac{P}{Q}\right\| \leq \frac{1}{Q}, x+\left[m^{\alpha}\right]=\left[y^{\alpha}\right], M^{1-\frac{1}{200}} k \leq m \leq M^{1-\frac{1}{200}}(k+1), \\
M \leq y, m \leq 2 M
\end{gathered}
$$

imply that

$$
y-A_{k}\left(1+\frac{10}{Q}\right) \leq\left(x_{P}+m^{\alpha}\right)^{1 / \alpha} \leq y+\frac{10 A_{k}}{Q} \text { for } P \neq 0,
$$

and one of the following estimates for $P=0$,

$$
y-A_{k}\left(1+\frac{10}{Q}\right) \leq\left(x_{0}+m^{\alpha}\right)^{1 / \alpha} \leq y+\frac{10 A_{k}}{Q}
$$


or

$$
y-A_{k}\left(1+\frac{10}{Q}\right) \leq\left(x_{1}+m^{\alpha}\right)^{1 / \alpha} \leq y+\frac{10 A_{k}}{Q}
$$

and consequently

$$
1_{A}\left(x+\left[m^{\alpha}\right]\right) \leq \begin{cases}\Psi_{k, P}^{\mathrm{u}}\left(\left(x_{P}+m^{\alpha}\right)^{1 / \alpha}\right) & \text { for } P \neq 0 \\ \Psi_{k, 0}^{\mathrm{u}}\left(\left(x_{0}+m^{\alpha}\right)^{1 / \alpha}\right)+\Psi_{k, 0}^{\mathrm{u}}\left(\left(x_{0}+1+m^{\alpha}\right)^{1 / \alpha}\right) & \text { for } P=0\end{cases}
$$

which implies (2.10). In order to obtain (2.12)-(2.14) we notice that a number $y \in \mathbb{N}$ satisfies $\left[y^{\alpha}\right]=x+\left[m^{\alpha}\right]=: z$ if and only if there exists $\theta \in[0,1)$ such that the first of the equalities below holds

$$
y=(z+\theta)^{1 / \alpha}=z^{1 / \alpha}+\frac{\theta}{\alpha z^{1-\frac{1}{\alpha}}}+\frac{\eta}{M^{2 \alpha-1}},|\eta|<1
$$

Thus,

$$
z^{1 / \alpha} \in\left(y-\frac{1}{\alpha z^{\frac{\alpha-1}{\alpha}}}-\frac{1}{M^{2 \alpha-1}}, y+\frac{1}{M^{2 \alpha-1}}\right) .
$$

Since by $(2.11), \Psi_{P}\left(m^{\alpha}\right) \neq 0$, we can write

$$
x+\left[m^{\alpha}\right]=x-\frac{P}{Q}+m^{\alpha}+\frac{\eta_{0}}{Q} \text { for } P \neq 0
$$

for some $\left|\eta_{0}\right|<1$. Then there exists $\eta_{1},\left|\eta_{1}\right| \leq 1$ such that

$$
z^{1 / \alpha}=\left(x_{P}+m^{\alpha}+\frac{\eta_{0}}{Q}\right)^{1 / \alpha}=\left(x_{P}+m^{\alpha}\right)^{1 / \alpha}+\frac{\eta_{1}}{Q M^{\alpha-1}} .
$$

Similar statement holds for $P=0$, possibly with $x$ replaced by $x_{1}$. Hence,

$$
\left(x_{P}+m^{\alpha}\right)^{1 / \alpha} \in\left(y-\frac{1}{\alpha z^{\frac{\alpha-1}{\alpha}}}-\frac{1}{M^{2 \alpha-1}}-\frac{1}{Q M^{\alpha-1}}, y+\frac{1}{M^{2 \alpha-1}}+\frac{1}{Q M^{\alpha-1}}\right) .
$$

In particular, for $M^{1-\frac{1}{200}} k<m<M^{1-\frac{1}{200}}(k+1)$ we have,

$$
\left(x_{P}+m^{\alpha}\right)^{1 / \alpha} \in\left(y-\frac{1}{\alpha z^{\frac{\alpha-1}{\alpha}}}-\frac{2}{Q M^{\alpha-1}}, y+\frac{2}{Q M^{\alpha-1}}\right) .
$$

Since for some $\eta_{2}$ with $\left|\eta_{2}\right| \leq 1$,

$$
\frac{1}{z^{\frac{\alpha-1}{\alpha}}}=\frac{1}{\left(x+m^{\alpha}\right)^{\frac{\alpha-1}{\alpha}}}+\frac{\eta_{2}}{M^{2 \alpha-1}},
$$

it follows from (2.15) and (2.16) that

$$
\begin{array}{r}
\left(x_{P}+m^{\alpha}\right)^{1 / \alpha} \in\left(y-\frac{1}{\alpha\left(x+m^{\alpha}\right)^{\frac{\alpha-1}{\alpha}}}-\frac{1}{M^{2 \alpha-1}}-\frac{2}{Q M^{\alpha-1}}, y+\frac{2}{Q M^{\alpha-1}}\right) \\
\subset\left(y-\frac{1}{\alpha\left(x+u_{k}^{\alpha}\right)^{\frac{\alpha-1}{\alpha}}}-\frac{3}{Q M^{\alpha-1}}, y+\frac{3}{Q M^{\alpha-1}}\right) .
\end{array}
$$

Thus we get (2.12)-(2.14). 
Expanding $\Psi_{k, P}^{\mathrm{u}}$ and $\Psi_{P}$ in (2.10) into the Fourier series we obtain,

$$
\begin{aligned}
& \mu_{M} * \tilde{\mu}_{M}(x) \\
& \leq \frac{1}{M^{2}} \sum_{P=0}^{Q-1} \sum_{k=M \frac{1}{200}}^{*} \sum_{\left(j_{1}, j_{2}\right) \neq(0,0)}^{2 M} c_{j_{1}}^{(k, P)} c_{j_{2}}^{(P)} \sum_{m=M^{1-\frac{1}{200}} k}^{M^{1-\frac{1}{200}}(k+1)} \varphi\left(\frac{m^{\alpha}}{M^{\alpha}}\right) \varphi\left(\frac{x+m^{\alpha}}{M^{\alpha}}\right) e^{2 \pi i f_{P}(m)} \\
& \quad+\frac{1}{M^{2}} \sum_{P=0}^{Q-1} \sum_{k=M \frac{1}{200}}^{2 M^{\frac{1}{200}}-1} \sum_{m=M^{1-\frac{1}{200}} k}^{M^{1-\frac{1}{200}}(k+1)} A_{k}\left(1+\frac{20}{Q}\right) \frac{1}{Q} \varphi\left(\frac{m^{\alpha}}{M^{\alpha}}\right) \varphi\left(\frac{x+m^{\alpha}}{M^{\alpha}}\right)=: I_{1}+I_{2},
\end{aligned}
$$

where $c_{j_{1}}^{(k, P)}$ and $c_{j_{2}}^{(P)}$ are Fourier coefficients of $\Psi_{k, P}^{\mathrm{u}}$ and $\Psi_{P}$, and moreover, we have used the fact that for $k M^{1-\frac{1}{200}} \leq m<(k+1) M^{1-\frac{1}{200}}$,

$$
0<c_{0}^{(k, P)}<A_{k}\left(1+\frac{22}{Q}\right) \leq \frac{1}{\alpha\left(x+m^{\alpha}\right)^{\frac{\alpha-1}{\alpha}}}+C M^{1-\alpha-\frac{1}{1000}}
$$

and $c_{0}^{(P)}=Q^{-1}$.

Let

$$
\rho_{M}(x)=\frac{1}{M^{2}} \sum_{m=1}^{\infty} \varphi\left(\frac{m^{\alpha}}{M^{\alpha}}\right) \varphi\left(\frac{x+m^{\alpha}}{M^{\alpha}}\right) \frac{1}{\alpha\left(x+m^{\alpha}\right)^{\frac{\alpha-1}{\alpha}}} .
$$

It is easy to see that

(1) $\left|\rho_{M}(x)-I_{2}\right| \leq C M^{-\alpha} Q^{-1}$,

(2) $\rho_{M}$ satisfies conditions (2.6).

Therefore, we have to show that for $1<\alpha<1+\frac{1}{1000}$ we have $\left|I_{1}\right| \leq M^{-\alpha-\frac{1}{1000}}$.

Notice that independently of $(k, P)$ and $(P)$, (see [10, chapter 1], for example)

$$
\sum_{\left|j_{1}\right| \geq M^{\alpha-1+\frac{1}{100}}}\left|c_{j_{1}}^{(k, P)}\right| \leq M^{-4} \text { and } \sum_{\left|j_{2}\right| \geq M \frac{1}{100}}\left|c_{j_{2}}^{(P)}\right| \leq M^{-4} .
$$

Therefore, it suffices to take $j_{1}, j_{2}$ satisfying assumptions of Corollary 2.3 in the sum defining $I_{1}$. Since also $\sum_{j_{1}, j_{2}}\left|c_{j_{1}}^{(k, P)}\right|\left|c_{j_{2}}^{(P)}\right| \leq C \log M$ (the proof is an easy exercise, $[10$, chapter 1]) we have

$$
\begin{aligned}
\left|I_{1}\right| & \leq \frac{Q M^{\frac{1}{200}} \log M}{M^{2}} \sup _{\left(j_{1}, j_{2}\right) \neq(0,0)}\left|S\left(j_{1}, j_{2}\right)\right| \\
& \leq Q M^{-\frac{1}{200}} M^{-1-\frac{1}{200}} M^{\frac{1}{200}} \log M \leq M^{-\alpha} M^{-\frac{1}{1000}} \text { for } 1<\alpha \leq 1+\frac{1}{1000},
\end{aligned}
$$

where the indices $j_{1}, j_{2}$ in the $\sup \left|S\left(j_{1}, j_{2}\right)\right|$ are as in Corollary 2.3. Hence, we obtain the upper bound in

$$
-C M^{-\alpha} M^{-\frac{1}{1000}} \leq \mu_{M} * \tilde{\mu}_{M}(x)-\rho_{M}(x) \leq C M^{-\alpha} M^{-\frac{1}{1000}} .
$$

To obtain the lower bound in (2.17) we repeat the proof with the following changes.

- We replace $\psi_{k, P}^{\mathrm{u}}$ in $(2.8)$ by the function $\psi_{k, P}^{1}$, defined below,

$$
\psi_{k, P}^{1}(x)= \begin{cases}F\left(\frac{Q}{A_{k+1}}\left(-A_{k+1}\left(1-\frac{20}{Q}\right)-x\right)\right) & \text { for }-\frac{1}{2} \leq x \leq-\frac{20 A_{k+1}}{Q} \\ F\left(\frac{Q}{A_{k+1}}\left(x+\frac{20 A_{k+1}}{Q}\right)\right) & \text { for }-\frac{20 A_{k+1}}{Q} \leq x \leq \frac{1}{2}\end{cases}
$$


where $F$ is defined in (2.7).

- Observe that similarly to $(2.12)$, for $k M^{1-\frac{1}{200}} \leq m \leq(k+1) k M^{1-\frac{1}{200}}$, the inequalities

$$
y-A_{k+1}\left(1-\frac{10}{Q}\right) \leq\left(x_{P}+m^{\alpha}\right)^{1 / \alpha} \leq y-\frac{10 A_{k+1}}{Q} \text { for } P \neq 0
$$

and $\left\|m^{\alpha}-\frac{P}{Q}\right\| \leq \frac{1}{Q}$ imply $x+\left[m^{\alpha}\right]=\left[y^{\alpha}\right]$. Hence, we have that for $P \neq 0$,

$$
\Psi_{k, P}^{1}\left(\left(x_{P}+m^{\alpha}\right)^{1 / \alpha}\right) \Psi_{P}\left(m^{\alpha}\right) \leq 1_{A}\left(x+\left[m^{\alpha}\right]\right) \Psi_{P}\left(m^{\alpha}\right) .
$$

- We do not take into account $P=0$ in the sumation in (2.10).

We leave the details for the reader.

In order to prove the estimate for $\rho_{M}(x)$ for $0<|x| \leq C M$ we argue as follows. Assume that the following equality holds

$$
x+\left[m^{\alpha}\right]=\left[(m+s)^{\alpha}\right] \text { where } s>0, x>0 .
$$

Then we have

$$
x-1 \leq(m+s)^{\alpha}-m^{\alpha} \leq x+1
$$

and hence

$$
c M^{\alpha-1} \leq c s M^{\alpha-1} \leq x \leq C s M^{\alpha-1} .
$$

Observe that for the increasing function $g(m)=(m+s)^{\alpha}-m^{\alpha}$ we have

$$
g(m+1)-g(m) \approx s M^{\alpha-2} \lesssim 1, \text { for }|x| \leq C M,
$$

and thus for a fixed $s$ there are at most $1+s^{-1} M^{2-\alpha} \approx M x^{-1}$ different consecutive values of $m$ for wchich (2.18) can hold. Since moreover, by (2.19), $s$ has to satisfy $s \approx x M^{1-\alpha}$, the total number of solutions of (2.18) is bounded from above by

$$
C M x^{-1}\left(x M^{1-\alpha}\right)=C M^{2-\alpha} .
$$

Hence we easily obtain $\rho_{M}(x) \leq C M^{-\alpha}$ for $0<|x|<C M$ and the lemma follows.

\section{Proof of Theorem 1.1}

Let

$$
M^{*} f(x)=\sup _{n>0}\left|\mu_{2^{n}} * f(x)\right| .
$$

With no loss of generality it suffices to show the weak type $(1,1)$ of $M^{*}$. We will use the argument of [5] and [11] adapted to our setting. Let $\lambda>0$ and $f \in \ell^{1}(\mathbb{Z})$. Let $N=2^{n}, n \in \mathbb{N}$. We consider the Calderón-Zygmund decomposition

$$
f=g+\sum f_{s, j}=\sum b_{s}+g,
$$

where $|g|<\lambda$ and $b_{s}$ contains terms $f_{s, j}$ supported by $Q_{s, j}$ with $\left|Q_{s, j}\right| \simeq 2^{\alpha s}$, $\sum_{s, j}\left|Q_{s, j}\right| \leq \lambda^{-1}\|f\|_{\ell^{1}}$ and $\left\|f_{s, j}\right\|_{\ell^{1}} \simeq \lambda\left|Q_{s, j}\right|$. We do not assume $\int f_{s, j}=0$, instead we decompose further each $b_{s}$ writing $b_{s}(x)=b_{s}^{(N)}(x)+B_{s}^{(N)}(x)+g_{s}^{(N)}(x)$, where $b_{s}^{(N)}(x)=\chi_{\left\{\left|b_{s}\right|>\lambda N\right\}}(x) b_{s}(x)$, and for $h_{s}^{(N)}(x)=b_{s}(x)-b_{s}^{(N)}(x)$ we have $B_{s}^{(N)}(x)=h_{s}^{(N)}(x)-g_{s}^{(N)}(x)$ and $g_{s}^{(N)}(x)=\sum_{j} \frac{\chi Q_{s, j}(x)}{2^{\alpha s}} \int_{Q_{s, j}} h_{s}^{(N)}$. Consequently,

$$
f=g+\sum_{s} g_{s}^{(N)}+\sum_{s} B_{s}^{(N)}+\sum_{s} b_{s}^{(N)} .
$$


Observe that $\frac{1}{2^{\alpha s}} \int_{Q_{s, j}}\left|h_{s}^{(N)}\right| \leq C \lambda$ and, since $Q_{s, j}$ are mutually disjoint, we get

$$
|g(x)|+\sum_{s}\left|g_{s}^{(N)}(x)\right| \leq C \lambda
$$

We have

$$
\left\{x: \mu_{N} *\left|b_{s}^{(N)}\right|(x)>0\right\}=\bigcup_{m \approx N}\left(\left[m^{\alpha}\right]+\left\{x:\left|b_{s}^{(N)}\right|(x)>0\right\}\right) .
$$

Thus,

$$
\begin{aligned}
\left|\left\{x: \mu_{N} *\left|b_{s}^{(N)}\right|(x)>0\right\}\right| & =\sum_{m \approx N}\left|\left\{x:\left|b_{s}^{(N)}\right|(x)>0\right\}\right| \\
& =N\left|\left\{x:\left|b_{s}^{(N)}\right|(x)>\lambda N\right\}\right| .
\end{aligned}
$$

Consequently (remember that $N=2^{n}$ ),

$$
\begin{aligned}
\sum_{s} \sum_{N-\text { dyadic }}\left|\left\{x: \mu_{N} *\left|b_{s}^{(N)}\right|(x)>0\right\}\right| & \leq \sum_{s} \sum_{N-\text { dyadic }} N\left|\left\{x:\left|b_{s}\right|(x)>\lambda N\right\}\right| \\
& \leq \sum_{s} \frac{1}{\lambda}\left\|b_{s}\right\|_{\ell^{1}} \leq \frac{1}{\lambda}\|f\|_{\ell^{1}} .
\end{aligned}
$$

Moreover, since for the fixed dyadic $N$ the supports of $B_{s}^{(N)}(x)$ are mutually disjoint, it is easy to see, for a fixed $x \in Q_{s_{0}, j_{0}}$,

$$
\begin{aligned}
\sum_{N \text { dyadic }} \sum_{s} N^{-1} B_{n-s}^{(N)}(x)^{2} \leq & \left(\sum_{\left\{N-\text { dyadic: } N \lambda \geq\left|b_{s_{0}}(x)\right|\right\}} N^{-1}\left|b_{s_{0}}(x)\right|^{2}\right) \\
& \left.+\lambda^{2} \sum_{N-\text { dyadic }} N^{-1} \chi_{\left\{\operatorname{supp} b_{s_{0}}\right\}}(x)\right) \\
\leq & C \lambda\left|b_{s_{0}}(x)\right|+\lambda^{2} \chi_{\left\{\operatorname{supp} b_{s_{0}}\right\}}(x) \\
\leq & C \lambda \sum_{s}\left(\left|b_{s}(x)\right|+C \lambda \chi_{\left\{\operatorname{supp} b_{s}\right\}}(x) .\right.
\end{aligned}
$$

Hence by (3.4), we have

$$
\lambda^{-2} \sum_{s} \sum_{N-\text { dyadic }} N^{-1}\left\|B_{n-s}^{(N)}\right\|_{\ell^{2}}^{2} \leq \frac{C}{\lambda}\|f\|_{\ell^{1}}
$$

We will use the following lemma,

Lemma 3.6. Let $N=2^{n}, n \in \mathbb{N}$. For sufficiently small $\delta>0$ we have the following estimates, see [5],

$$
\left\|\mu_{N} * B_{n-s}^{(N)}\right\|_{\ell^{2}}^{2} \leq C \lambda\left\|B_{n-s}^{(N)}\right\|_{\ell^{1}} 2^{-\delta s}+2^{-n}\left\|B_{n-s}^{(N)}\right\|_{\ell^{2}}^{2}
$$

and for $s_{1}>s_{2}$,

$$
\left|\left\langle\mu_{N} * B_{n-s_{1}}^{(N)}, \mu_{N} * B_{n-s_{2}}^{(N)}\right\rangle\right| \leq C \lambda\left\|B_{n-s_{2}}^{(N)}\right\|_{\ell^{1}} 2^{-\delta s_{1}} .
$$


Proof. By Lemma 2.5,

$$
\mu_{N} * \tilde{\mu}_{N}(x)=C \rho_{N}(x)+2^{-n} \delta_{0}(x)+O\left(2^{-n\left(\alpha+\frac{1}{1000}\right)}\right),
$$

where $\rho_{N}$ satisfies

$$
\left|\rho_{N}(x)\right| \leq \frac{C}{N^{\alpha}}=\frac{C}{2^{\text {nd }}} \text { and } \rho_{N}(0)=0 .
$$

Moreover, for $|x|>C M$ and $|x+h|>C M$,

$$
\left|\rho_{N}(x+h)-\rho_{N}(x)\right| \leq \frac{C}{2^{n \alpha}} \frac{|h|}{2^{n \alpha}} .
$$

Let for $s \leq n, \operatorname{supp} B_{s}^{(N)} \subset\left[0,4 \cdot 2^{n \alpha}\right]$ and $\operatorname{supp} \rho_{n} \subset\left[0,4 \cdot 2^{n \alpha}\right]$. Denote by $F_{s, j}$ the restriction of $B_{s}^{(N)}$ to $Q_{s, j}$ By the estimate $\left\|B_{s}^{(N)}\right\|_{\ell^{1}} \leq \lambda 2^{s \alpha}$ we have,

$$
\begin{aligned}
A:= & \left|\left\langle\mu_{N} * B_{n-s_{1}}^{(N)}, \mu_{N} * B_{n-s_{2}}^{(N)}\right\rangle\right|=\left|\left\langle\mu_{N} * \tilde{\mu}_{N} * B_{n-s_{1}}^{(N)}, B_{n-s_{2}}^{(N)}\right\rangle\right| \\
\leq & 2^{-n \alpha} 2^{-\frac{n}{1000}}\left\|B_{n-s_{1}}^{(N)}\right\|_{\ell^{1}}\left\|B_{n-s_{2}}^{(N)}\right\|_{\ell^{1}}+\left\langle\sum_{j_{1}}\left|\rho_{n} * F_{n-s_{1}, j_{1}}\right|,\left|B_{n-s_{2}}^{(N)}\right|\right\rangle \\
& +N^{-1}\left|\left\langle B_{n-s_{1}}^{(N)}, B_{n-s_{2}}^{(N)}\right\rangle\right| .
\end{aligned}
$$

Observe that for $s_{1} \neq s_{2}$ the supports of $B_{n-s_{1}}^{(N)}, B_{n-s_{2}}^{(N)}$ are disjoint and consequently the third summand is equal to zero. Consider the second summand in (3.10). By the regularity estimate (3.9) and the fact that $\int F_{n-s, j}=0$ we get in a standard way

$$
\left|\rho_{N} * F_{n-s_{1}, j}(x)\right| \leq \frac{2^{\left(n-s_{1}\right) \alpha}}{2^{n \alpha}} \frac{\left\|F_{n-s_{1}, j}\right\|_{\ell^{1}}}{2^{n \alpha}}
$$

for $\left|x-x_{s_{1}, j}\right|>C N+C 2^{\left(n-s_{1}\right) \alpha}$, where $x_{s_{1}, j}$ denotes the center of $Q_{s_{1}, j}$. Moreover, for any $x,\left|\rho_{N} * F_{n-s_{1}, j}(x)\right| \leq N^{-\alpha}\left\|F_{n-s_{1}, j}\right\|_{\ell^{1}} \leq \lambda N^{-\alpha} 2^{\left(n-s_{1}\right) \alpha}$. Consequently, we have

$$
\begin{aligned}
\sum_{j}\left|\rho_{N} * F_{n-s_{1}, j}(x)\right| \leq & \sum_{\left\{j:\left|x-x_{s_{1}, j}\right| \leq C N+C 2^{\left(n-s_{1}\right) \alpha}\right\}} 2^{-n \alpha}\left\|F_{n-s_{1}, j}\right\|_{\ell^{1}} \\
& +2^{-s_{1} \alpha} \sum_{\left\{j:\left|x-x_{s_{1}, j}\right|>C N+C 2^{\left(n-s_{1}\right) \alpha}\right\}} 2^{-n \alpha}\left\|F_{n-s_{1}, j}\right\|_{\ell^{1}} \\
\leq & C \lambda \max \left\{N^{1-\alpha}, 2^{-s_{1} \alpha}\right\}+C \lambda 2^{-s_{1} \alpha} \leq C \lambda 2^{-s_{1} \delta} .
\end{aligned}
$$

Thus we estimate the second summand in (3.10) by

$$
\left\|B_{n-s_{2}}^{(N)}\right\|_{\ell^{1}}\left\|\sum_{j}\left|\rho_{N} * F_{n-s_{1}, j}\right|\right\|_{\ell^{\infty}} \leq C \lambda 2^{-s_{1} \delta}\left\|B_{n-s_{2}}\right\|_{\ell^{1}} .
$$

Finally, we get

$$
A \leq C \lambda 2^{-s_{1} \delta}\left\|B_{n-s_{2}}\right\|_{\ell^{1}}+C \lambda\left\|B_{n-s_{2}}\right\|_{\ell^{1}} 2^{-\frac{n}{1000}} \leq C \lambda\left\|B_{n-s_{2}}\right\|_{\ell^{1}} 2^{-\delta s_{1}} .
$$

The assumption $\operatorname{supp} B_{n-s}^{(N)} \subset\left[0,4 \cdot 2^{n \alpha}\right]$ can be removed in a standard way. The estimates (3.7), (3.8) follow. 
Now we are ready to give

Proof of Theorem 1.1. Using Lemma 3.6 we have,

$$
\begin{aligned}
& \lambda^{2}\left|\left\{x: \max _{N}\left|\sum_{s \geq 0} B_{n-s}^{(N)} * \mu_{N}(x)\right| \geq c \lambda\right\}\right| \leq \sum_{x} \max _{N}\left|\sum_{s \geq 0} B_{n-s}^{(N)} * \mu_{N}(x)\right|^{2} \\
& \leq \sum_{x} \sum_{N}\left|\sum_{s \geq 0} B_{n-s}^{(N)} * \mu_{N}(x)\right|^{2} \\
& \leq \sum_{N, s \geq 0}\left\|\mu_{N} * B_{n-s}^{(N)}\right\|_{\ell^{2}}^{2}+2 \sum_{N, s_{1}>s_{2}}\left|\left\langle\mu_{N} * B_{n-s_{1}}^{(N)}, \mu_{N} * B_{n-s_{2}}^{(N)}\right\rangle\right| \\
& \leq \sum_{N, s \geq 0} C \lambda\left\|B_{n-s}^{(N)}\right\|_{\ell^{1}} 2^{-\delta s}+2^{-n}\left\|B_{n-s}^{(N)}\right\|_{\ell^{2}}^{2}+2 \sum_{N, s_{1}>s_{2}} C \lambda\left\|B_{n-s_{2}}^{(N)}\right\|_{\ell^{1}} 2^{-\delta s_{1}} \\
& \leq C \lambda\|f\|_{\ell^{1}},
\end{aligned}
$$

where the second summand in the last inequality is estimated by (3.5).

By (3.1) we have,

$$
\begin{aligned}
& \left\{\sup _{N}\left|\mu_{N} * f(x)\right|>4 C \lambda\right\} \subset\left\{\sup _{N}\left|\mu_{N} *\left(|g|+\sum_{s}\left|g_{s}^{(N)}\right|\right)(x)\right|>C \lambda\right\} \\
& \cup\left\{\sup _{N}\left|\mu_{N} *\left(\sum_{s>0}\left|B_{n-s}^{(N)}\right|\right)(x)\right|>C \lambda\right\} \cup\left\{\sup _{N}\left|\mu_{N} *\left(\sum_{s>0}\left|B_{n+s}^{(N)}\right|\right)(x)\right|>C \lambda\right\} \\
& \cup\left\{\sup _{N} \sum_{s} \mu_{N} *\left|b_{s}^{(N)}\right|>C \lambda\right\}=: S_{1} \cup S_{2} \cup S_{3} \cup S_{4} .
\end{aligned}
$$

In the above sum, by (3.2), the first set is empty if the constant $C$ is sufficiently large. Since $\operatorname{supp} \mu_{N} * B_{s}^{(N)} \subset \cup_{s, j} Q_{s, j}^{* * *}$ for $s \geq n$, the set $S_{3}$ is a subset of $\cup_{s, j} Q_{s, j}^{* * *}$ and consequently $\left|S_{3}\right| \leq\left|\cup_{s, j} Q_{s, j}^{* * *}\right| \leq C \sum_{s, j}\left|Q_{s, j}\right| \leq \frac{C}{\lambda}\|f\|_{\ell^{1}}$. Moreover by (3.11) we have $\left|S_{2}\right| \leq \frac{C}{\lambda}\|f\|_{\ell^{1}}$. The set $S_{4} \subset \bigcup_{N, s}\left\{\mu_{N} *\left|b_{s}^{(N)}\right|(x)>0\right\}$. Hence by (3.3) $\left|S_{4}\right| \leq \sum_{N, s}\left|\left\{\mu_{N} *\left|b_{s}^{(N)}\right|(x)>0\right\}\right| \leq \frac{C}{\lambda}\|f\|_{\ell^{1}}$. The theorem follows.

\section{Acknowledgements.}

The authors want to thank to J. Rosenblatt for communicating [4] and other related papers, and to M. Christ for a discussion on the subject of the paper.

\section{References}

[1] M. Boshernitzan, G. Kolesnik, A. Quas, and M. Wierdl, Ergodic averaging sequences, J. Anal. Math. 95 (2005), 63-103.

[2] J. Bourgain, On the maximal ergodic theorem for certain subsets of the integers, Israel J. Math. 61 (1988), no. 1, 39-72.

[3] — Pointwise ergodic theorems for arithmetic sets, Inst. Hautes Études Sci. Publ. Math. (1989), no. 69, 5-45.

[4] Z. Buczolich, Universally $L^{1}$ good sequences with gaps tending to infinity, arXiv:math.

[5] M. Christ, Weak type $(1,1)$ bounds for rough operators, Ann. of Math. (2) 128 (1988), no. 1, $19-42$.

[6] J.-M. Deshouillers, Problème de Waring avec exposants non entiers, Bull. Soc. Math. France 101 (1973), 285-295.

[7] — Un problème binaire en théorie additive, Acta Arith. 25 (1973-1974), 393-403. 
[8] C. Fefferman, Inequalities for strongly singular convolution operators, Acta Math. 124 (1970), 9-36.

[9] S. A. Gritsenko, Three additive problems, Izv. Ross. Akad. Nauk Ser. Mat. 56 (1992), no. 6, 1198-1216. Translation in Russian Acad. Sci. Izv. Math. 41 (1993), no. 3, 447-464.

[10] A. A. Karatsuba, Basic analytic number theory, Springer-Verlag, Berlin (1993), ISBN 3-54053345-1.

[11] A. Seeger, T. Tao, and J. Wright, Pointwise convergence of lacunary spherical means, in Harmonic analysis at Mount Holyoke (South Hadley, MA, 2001), Vol. 320 of Contemp. Math., 341-351, Amer. Math. Soc., Providence, RI (2003).

[12] J. G. van der Corput, Neue zahlentheoretische Abschätzungen, Math. Z. 29 (1929), no. 1, $397-$ 426.

Institute of Mathematics, Wroclaw University, Plac Grunwaldzki 2/4, 50-384 WroCLAW, POLAND

E-mail address: urban@math.uni.wroc.pl

SAME ADDRESS IN WROCLAW

E-mail address: zenek@math.uni.wroc.pl 